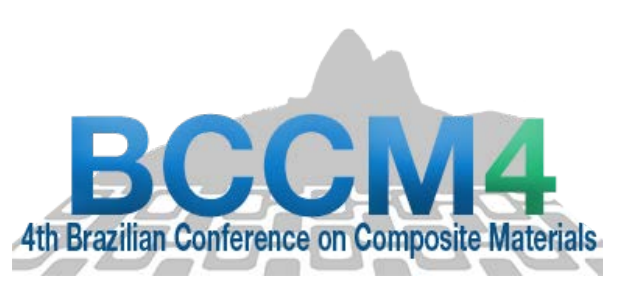

\title{
KRAFT LIGNIN FROM PAPER MILL LIGNOBOOST® PROCESS - A PROMISE TO LOW COST GREEN CARBON FIBER
}

\author{
Katia S. D. Nunes $^{(1)}$ and Luiz C. Pardini (2)
}

(1) Departamento de Química dos Materiais em Ciências e Tecnologias Espaciais (CTE-Q) -ITA - Instituto Tecnológico de Aeronáutica - São José dos Campos, Brazil

(2) Departamento de Ciência e Tecnologia Aeroespacial - DCTA - Instituto de Aeronáutica e Espaço-IAE, Divisão de Materiais (AMR) - São José dos Campos, Brazil

https://doi.org/10.21452/bccm4.2018.14.06

\begin{abstract}
The application of lignins in biodegradable/biobased materials is receiving increasing attention as the world looks for alternatives to petrochemicals. A new industrial method used to obtain higher purity and low cost lignin is now possible by the LignoBoost ${ }^{\circledR}$ process. The kraft lignin from this process has high carbon content level and opened a new research line for the production of products with high economic value like low cost carbon fiber. Kraft lignin is a paper mill waste with very low production costs that could open new possibilities for the lignin applications. In this work, three different types of lignin were compared with a regular carbon fiber precursor in order to verify their potential as alternative low cost precursor materials. The key aspect to be considered in the assessment of potential use of the material in the production of precursors is the carbon content level since it is the foremost constituent element of carbon-based materials.
\end{abstract}

\section{INTRODUCTION}

The use of biologically derived polymers (bio macromolecules) is emerging as an important component for economic development. By transforming forest and agricultural feedstock's, a new class of renewable, biodegradable and biocompatible materials (biomaterials) is being introduced [1, 2]. Emerging applications for bio macromolecules range from packaging and industrial chemicals, to producing 'green' materials with unique physical and functional properties, the processes used to create bio-based materials lead to new manufacturing opportunities that minimize energy consumption and waste [1]. Recently, environmental concerns have sparked interest in utilizing biodegradable and bio-derived materials in various industrial 
fields. Particularly, the utilization of plant-derived materials such as agricultural residues and forest products has received increasing attention. Biomass based fuels have found renewed interest because of the rising cost of oil. Common sources of biomass include wood, agricultural crops and their residues. Although more research is being done on wood as a fuel, there is a growing interest in alternatives $[2,4]$.

Particularly, lignin has gained interest as a source of fuel and new materials. The main reason for this trend is a growing concern for more sustainability, which results in an increased usage of renewable materials to replace petrochemicals [7]. Lignin is one of the most abundant bio macromolecules existing in the plant kingdom. An enormous amount of lignin is produced as a by-product of the pulp and paper industry. As a result, a number of systems have been proposed for the utilization of lignin as a renewable polymeric material. However, lignin utilization is still limited, with less than $2 \%$ of the lignin produced being used in high value commercial applications. Lignin is a relatively inexpensive and as a precursor for low cost carbon fibers could be very attractive. Lignin is a high molecular weight polyaromatic macromolecule with a reported total worldwide production of approximately 26 million tons/year. This by-product of 'wood-free' paper making, it is predominately burnt in a chemical recovery process as a fuel source. However, as a fuel it is relatively inefficient, producing less than about $1 / 2$ as much energy per kilogram as middle distillate (diesel, jet and boiler) fuels. Nonetheless, lignin combustion plays a critical role in the papermaking chemical recovery process and it is vital to that industry. However, an everincreasing number of paper mills have become chemical recovery limited; if paper production is to maximize, the by-product lignin can no longer be used in its traditional role as a fuel [2, 3, 7]. Biodegradability of biopolymers is the base for their applications, however, their processability, performance and in particular price as compared to synthetic petrochemical-based polymers are of extreme importance. To this end, materials such as agricultural residues and/or bioprocessing byproducts are obvious sources of low cost biomacromolecule materials [1, 7].

\subsection{LIGNIN STRUCTURE}

Lignin is a complex three-dimensional network polymer, as showed in Figure 2. Lignin behaves as a continuous matrix component in plant cell walls, providing mechanical strength and structural support [1]. The lignin binds fibers together to form a strong and tough matrix of plants and provides mechanical support to the plant vessels for the transportation of water and nutrients [6]. Depending on the wood species, e.g., softwood (conifers) or hardwood (angiosperms), lignins can contain predominately glycerol-aryl ether linkages, but there are several types of C-C bonds which likely serve as crosslinks between relatively short, linear chains of phenyl propane units [5, 9]. There is no method to isolate lignin from plants in the native form, chemical and physical modifications are unavoidable during lignin isolation. Therefore, the chemical structure and thermal behaviour of lignin is strongly dependent on the isolation method used. Isolated lignin displays both thermoplastic and thermosetting behaviour. The thermal properties of lignin are usually explained in association with its chemical structure, i.e., molecular weight, degree of condensation, and chemical modification during the preparation process. However, noncovalent interactions, such as hydrogen bonding, will also affect the thermal properties of lignin; strong interactions will reduce the thermal molecular motion of the lignin molecules $[5,7,9]$. 


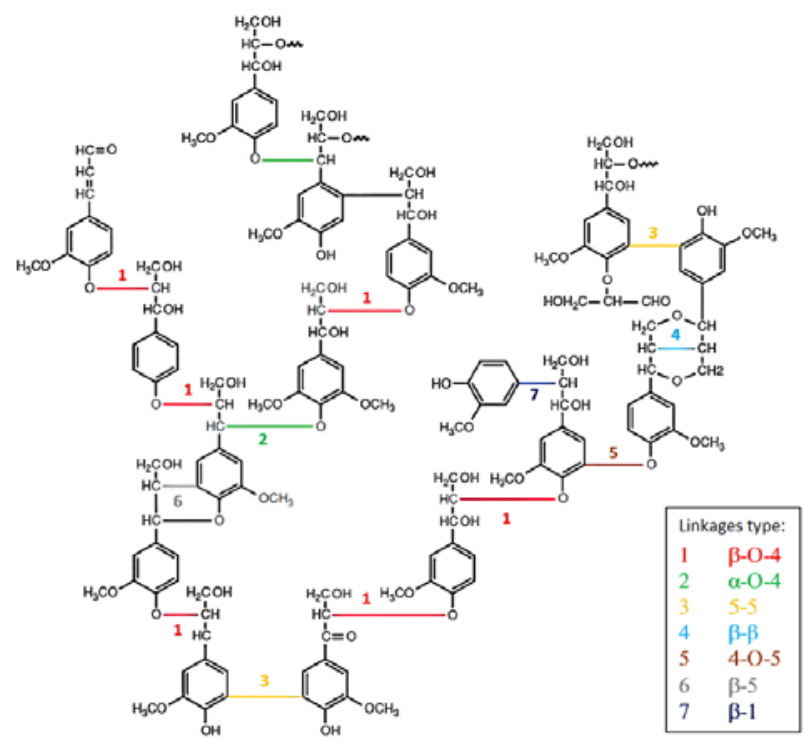

Figure 1: Model structure from hardwood lignin [9]

The traditional process of lignin precipitation and separation from kraft black liquors causes severe problems, related to complete or partial plugging of the filter cake and/or of the filter medium. Plugging of these types of filter cakes results in an extremely low level of the washing liquor flow through the cake and, consequently, to the need for extremely large filter areas. Partial plugging of the filter cake also leads to high levels of impurities in the lignin. It has been shown that these negative effects on the filtration process are caused by changes in lignin solubility, due to an excessive $\mathrm{pH}$ level and to the ionic strength gradients in the lignin filter cake during the washing process. Such changes result in restructuring the lignin particles, e.g. returning to a colloidal state, or in dissolution and reprecipitation of lignin in the filter medium $[8,14]$.

\subsection{THE LIGNOBOOST ${ }^{\circledR}$ PROCESS}

The development of the LignoBoost ${ }^{\circledR}$ process was carried out to reduce costs and impurities from kraft lignin [3]. Furthermore, full-scale combustion trials were carried out to evaluate the effects of firing the lignin bio fuel product in two different types of boilers and in a limekiln generation. One way of exploiting the energy surplus of a modern kraft pulp mill is to extract lignin from the black liquor. This gives the pulp mill a great opportunity to develop new economical revenues when the new by-product, lignin, is commercialised or used within the pulp mill to reduce production costs [8]. Lignin extraction has the additional advantage of providing an incremental capacity in the chemical recovery area, so that it can be used to off-load the recovery boiler or to avoid expansion, when pulp production is increased [7, 8]. The LignoBoost ${ }^{\circledR}$ process therefore makes it possible to extract lignin efficiently from the black liquor in kraft mills. The major advantages, compared to the previous technology, are the following: 1- the filter area and the volume of acidic washing water can be kept at lower values, resulting in lower investment costs, 2- the addition of sulfuric acid can be also kept at a lower level, resulting in lower operational costs, 3- the yield of lignin is higher, 4- the lignin has a lower ash and carbohydrate content, 5- the lignin has a higher content of dry solids [7]. The main idea is that kraft lignin processing, precipitated from black liquor produce a solid biofuel with high energy density and low ash 
content, to use the dry lignin powder as a biofuel in the lime kiln and swap fossil fuel. So, a lignin without chlorides and lower ash content could be use in other burners/boilers, where fossil fuel is normally used, boilers in which corrosion from chlorides is a problem or, generally, in fluidised bed boilers frequently used for biomass combustion [8].

In the LignoBoost ${ }^{\circledR}$ Process, a stream of black liquor is taken from the black liquor evaporation plant (Fig. 2), then lignin is precipitated by acidification (the preferred acid is $\mathrm{CO}_{2}$ ) and filtered (“chamber press filter 1", Fig. 2). Instead of washing lignin immediately after filtration, as in traditional processes, the filter cake is re-dispersed and acidified (“cake re-slurry”, Fig. 2) [8].

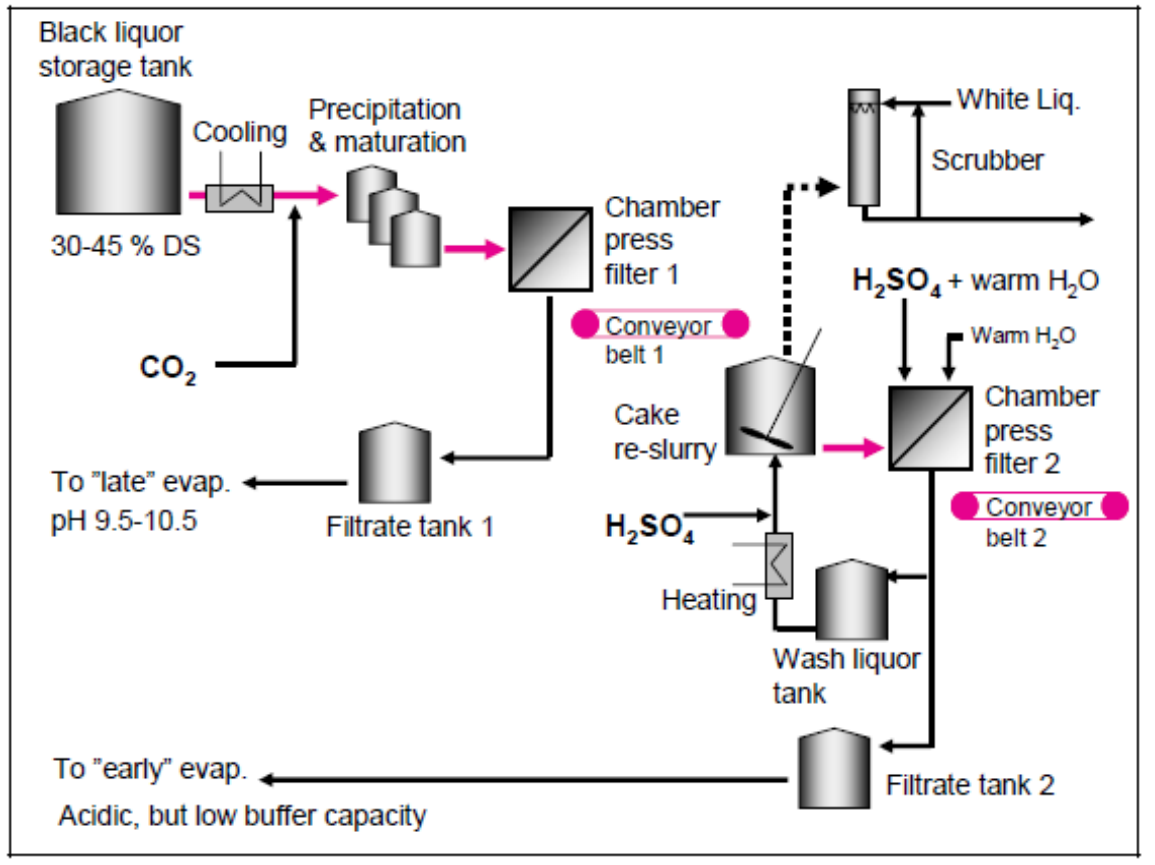

Figure 2: General layout of the LignoBoost ${ }^{\circledR}$ lignin removal process (post-treatment, drying and pulverizing are excluded) [8].

The resulting slurry is then filtered and washed by means of displacement washing ("chamber press filter 2”, Fig. 2). When the filter cake is re-dispersed in a liquid, at $\mathrm{pH}$ level and temperature values approximately equal to those of the final washing liquor, the concentration gradients during the washing stage will be low. The change in the $\mathrm{pH}$ level, most of the change in ionic strength and any change in lignin solubility will then take place in the slurry, and not in the filter cake or in the filter medium during washing [8].

\subsection{LIGNIN AS A CARBON FIBER PRECURSOR}

Today approximately 95\% of all carbon fiber uses polyacrylonitrile (PAN) as a precursor [10]. The high cost of carbon fiber production limits its widespread use. The basic difficulty is to combine precursor cost, yield and processing cost. Efforts to decrease the costs of carbon fiber and environmental concerns production are ongoing and kraft lignin is an attempt on this way due to being a biodegradable and bio-derived waste residue $[3,10]$.

With the development of the LignoBoost ${ }^{\circledR}$ process and the obtation of higher quality kraft lignin, the possibility of a new line of lignin use was opened. The kraft lignin contains more carbon compared to any other bio fuels showing great potential in the manufacture of carbon fiber precursor [8]. 
Due to the great potential as a precursor for carbon fiber, a lot attempts are being made with lignins. The domestic paper industry, could annually yield sufficient precursor for the present worldwide carbon fiber production needs without affecting paper mill operations. Carbon fibers have been manufactured from lignin. Kayacarbon lignin carbon fiber, was first developed and made commercially available by Nippon Kayaku Co. on a pilot scale. The process involved carbonization of dry spun fibers from lignin dissolved in alkali solution with poly(vinyl alcohol) added as a plasticizer. Sudo and co-workers showed that lignin could be converted into a molten viscous material with suitable properties for thermal spinning by hydrocracking, phenolation, or hydrogenolysis followed by heat treatment in vacuum. These modification methods seem to induce flow by remove as possible hydroxyl and hydroxy methyl functional groups from the lignin. The resulting carbon fibers showed superior properties to those of the Kayacarbon. More recently, Sano et al. have produced carbon fibers with properties suitable for midrange markets from organosolv lignin obtained by aqueous acetic pulping. Unfortunately in each of previous systems utilizing lignin, concerning production costs exists. Either plasticization or lignin modification was required, or no commercially available carbon fibers from commercially available lignins were used [3].

\section{EXPERIMENTAL PROCEDURE}

Kraft Lignin from LignoBoost ${ }^{\circledR}$ process was analyzed by elementary analyses. For content quantification of $\mathrm{C}, \mathrm{N}, \mathrm{H}, \mathrm{S}$ and $\mathrm{O}$ in lignin samples it was used a calibration curve with minimum requirements for the correlation coefficient $\left(\mathrm{r}^{2}\right)$ greater than 0.99 for the reference analytical standard. The detection mode for $\mathrm{N}$ was thermal conductivity and to $\mathrm{S}, \mathrm{C}$ and $\mathrm{H}$ the detection mode was IR. Oxygen gas was used to burning all the raw materials and Helium was used as gas drag. To elementary analysis of $\mathrm{C}, \mathrm{N}$ and $\mathrm{H}$ was done by weighting $0.100 \mathrm{~g}$ of raw materials and completely incinerated at $1050{ }^{\circ} \mathrm{C}$. The oxygen content was obtained by difference according to the guidelines of Bech et al. and Protásio et al. [11, 12]. The elementary analysis for sulfur was done weighting $\pm 0.1000 \mathrm{~g}$ of the sample in a porcelain crucible by complete incineration of the sample at $1450{ }^{\circ} \mathrm{C}$. Detection was accomplished by IR mode.

\section{MATERIALS}

The kraft lignin used in this work is a hardwood lignin obtained from eucalyptus, by using LignoBoost ${ }^{\circledR}$ process [13], from Fibria S.A.

A Sigma Aldrich lignin was analysed for comparation. It is a commercial kraft lignin obtained from Sigma Aldrich Brasil.

The analytical standard used for quantification level of the elements was EDTA (LECO, USA), with the following contents: carbon (\%): 41.07 \pm 0.13 ; hydrogen (\%): 5.5 \pm 0.03 ; nitrogen (\%): $9.56 \pm 0.02$. The analytical standard used for quantification level of contained sulfur was petroleum coke (LECO, USA), with the following contents: sulfur (\%): 5.67 \pm 0.14 ; volatile (\%): $13.0 \pm 0.70$; ash (\%): 0.27 \pm 0.06 ; were conduced on a CHN 628 (LECO) for Module S and 628S (LECO) for module CHN. 


\section{RESULTS AND DISCUSSION}

The results from elementary analyses for the samples of different lignins and PAN polymer are shown in Table 1. The comparison between carbon content shows that kraft lignin from LignoBoost ${ }^{\circledR}$ process has very high percentage of carbon in its chemical constitution. It is also possible to observe that the level of carbon content from kraft lignin is very close to the level found in PAN, which is the main and widely employed material for the production of carbon fiber. Elementary analysis of kraft lignin obtained by INVENTIA, also exhibits similar results with the ones found in this work [8]. On the other hand, the carbon content for a lignin available from Sigma Aldrich shows values lower than the ones from INVENTIA lignins. Most probably Sigma Aldrich lignin is also obtained by the kraft process, which in this case, resulted in low carbon content. The sulphur content at the analysed lignins are close, $1 \%-3 \%$, which is typical for paper mill plants [8]. Sigma Aldrich lignin shows slightly higher levels of sulphur when compared to the other lignins. Still for the Sigma Aldrich lignin, the level of oxygen content is remarkably higher than the other presented samples. High oxygen content on lignin brings problems on the next production processes due to molecular interaction driven by oxygen and hydrogen bonds. It gives lower mobility and higher Tg to lignin. Many attempts have been described to eliminate hydroxyl $(-\mathrm{OH})$ and hydroxyl methyl functional groups $(-\mathrm{CH} 2-\mathrm{OH})$ from lignin. These studies on lignin processes are intended to produce carbon fibers with superior properties, with target on midrange markets [3].

Table 1: Elementary analyses to lignin and PAN.

\begin{tabular}{cccccc}
\hline Sample & N (\%) & C (\%) & H (\%) & O (\%) & S (\%) \\
\hline INVENTIA lignin* & 0.10 & 65.10 & 5.80 & 26.10 & 2.50 \\
kraft lignin & 0.15 & 62.74 & 5.86 & 26.90 & 2.19 \\
Sigma Aldrich lignin & 0.12 & 46.7 & 5.21 & 44.36 & 3.61 \\
PAN (Radici Fibras) & 26.3 & 66.4 & 5.80 & 1.4 & 0.10 \\
* Source: [8]. & & & & &
\end{tabular}

\section{CONCLUSION}

This work focused on the review of one relevant chemical characteristic of the lignin as a raw material for carbon based precursors. The results of the elementary analyses for kraft lignin from FIBRIA and Sigma Aldrich and for PAN were reviewed and compared with available data from literature. Values of carbon content in the range of $60-65 \%$ are typical from the LignoBoost ${ }^{\circledR}$ process. It was shown that its carbon content is close to the carbon content of PAN polymer, which is the classical and most important industrial precursor used to obtain carbon fiber. The carbon content from kraft lignin obtained from FIBRIA meets the standard quality for use as a carbon based precursor. 
$4^{\text {th }}$ Brazilian Conference on Composite Materials. Rio de Janeiro, July $22^{\text {nd }}-25^{\text {th }}, 2018$

\section{ACKNOWLEDGEMENTS}

This work was main possible due to the support from FIBRIA, CAPES, Quimlab and DCTA.

\section{REFERENCES}

[1] Kadla, J. F. and Kubo, S., 'Lignin-based polymer blends: Analysis of intermolecular interactions in lignin - synthetic polymer blends', Composites: Part A, 35 (2004) 395-400.

[2] Kubo, S. and Kadla, J. F., 'Lignin-based carbon fibers: Effect of synthetic polymer blending on fiber properties', Journal of Polymers and the Environment, 13 no. 2 (2005) 97-105.

[3] Kadla, J. F., Kubo, S., Venditti, R. A., Gilbert, R.D., Compere, A.L., Griffith, W., 'Lignin-based carbon fibers for composite fiber applications', Carbon, 40 (2002) 2913-2920.

[4] Chandrasekaran, S.R., Hopke, P.K., Kinetics of switch grass pellet thermal decomposition under inert and oxidizing atmospheres", Bioresource Technology,125 (2012) 52-58.

[5] Kubo, S., and Kadla, J.F., 'Hydrogen Bonding lignin: A Fourier transform infrared model compound study', Biomacromolecules, 6 (2005) 2815-2821.

[6] Pan, X., Saddler, J.N., 'Éffect of replacing polyol by organosolv and kraft lignin on the property and structure on rigid polyuretane foam' Biotechnology for Biofuels, 6:12 (2013) 1-10.

[7] Brodin, I., 'Chemical Properties and thermal behaviour of kraft lignins' Thesis, KTH Royal Institute of Technology, School of Chemical Sciences and Engineering, Division of Wood Chemistry and Pulp Technology, (Stockolm 2009).

[8] Tomani, Peter, 'The LignoBoost process', Cellulose Chemistry and Technology, 44(1-3), (2010) 53-58.

[9] Laurichesse, S., Averus, L., 'Chemical modification of lignins. Towards biodased polymers.' Progress in Polymer Science, 39, 7, (2014) 1266-1290.

[10]Huson, M.G., Church, J.S., Kafi, A.A., Woodhead, A.L., Khoo, J., Kiran, M.S.R.N., Bradly, J.E., Fox, B.L., 'Heterogeneity of carbon fibre', Carbon, 68, (2014) 240-249.

[11]T. P. Protásio, et all., 'Braziliam Journal of Forest Research - Pesquisa Florestal Brasileira', Colombo 31, 66, 113 (abr./jun. 2011).

[12]N. Bech, P. A. Jensen, K. Dam-Johansen, Biomass and Bioenergy 33, Issue 3, (March 2009) 534537.

[13]http://compositesmanufacturingmagazine.com/2016/01/state-of-the-composites-industry-lucintel2016/6/

[14]Patent applications 1794363, 1797236 and PCT/SE 2008/000142 